\title{
Ticket scalpers
}

\section{What would you do if you could publish only 20 papers throughout your career?}

$\mathbf{T}$ here are researchers who think that something is seriously wrong with the way science is published.

Some complain about power: "Editors of 'high-profile" journals have too much power over scientists, because their decisions profoundly affect one's chances to get a grant/job/ tenure." However, with a number of excellent journals to choose from, an important paper should find a good home in one of them, despite any poor editorial decisions from the others. Besides, high-profile status is ultimately conferred to a journal by the community, and publications that don't do a good job of filtering scientific information (an important raison d'etre for professional editors) do pay the price.

Others complain about money: "Publishing firms make a fortune selling back to universities the papers that scientists produce." The open-access movement stems at least in part from this complaint. So much ink has been spilled over this argument that it is hard to say anything new. Instead, let's acknowledge that if there are so many journals on the market, it's because of the tendency of authors to submit very incremental papers-what one could call 'minimal publishable units'. There are as many scientists who 'slice the salami' as thin as possible as there are specialized journals willing to publish their work.

It's certainly possible to argue that the proliferation of papers also results from an explosion of research disciplines, each of which needs its own set of journals, and from the need the community has for the independent replication of published results. One could also say that trainees need papers to advance to the next stage of their careers and that researchers need papers to show funding agencies where their money has been going.

For the sake of argument, though, let's agree that there are problems about cost and power in scientific publishing that need to be fixed. So, here's a bold proposal to reduce both the number of publications that your library has to pay for and the influence of editors on what is granted visibility in highprofile journals-let's set a limit on the number of papers that scientists can publish during their careers.

These are the basic rules: whenever you get your first academic job (that is, the first lab of your own), you get 20 tickets.
Every time you publish a paper, you hand over one of them. Once you run out of tickets, your publishing days are over. As simple as that.

If we adopted this model, many articles reporting incremental advances would no longer be written, and many specialized journals would disappear. And with far fewer papers to read, each one reporting a much more complete piece of research, search committees or funding bodies could directly evaluate the work of a given scientist, instead of (as is often the case) leaning on surrogate indicators such as a journal's impact factor or number of citations.

At the extreme, we might not even need journals (and editors) anymore; everything would be published in preprint servers like those used by physicists, and the community would simply evaluate and rank the different contributions as they become available. This way, the whole community could act as reviewers, doing away with the existing peer-review process. This is somewhat reminiscent of what some websites are already trying to do, so far with limited success. But if everybody agreed to publish just 20 papers to keep the size of the literature manageable, then the journal of the future might conceivably be a preprint server.

As this model is adopted, it will certainly need some tweaking. Maybe a particularly seminal paper would be exempt from ticket usage. Would review articles require a ticket? Maybe a much coveted award could come with half an extra ticket, and a very competitive postdoctoral fellowship could get you one-fourth of a ticket. If you collaborate with another lab, you could be sole senior author of a paper at the expense of one ticket or, alternatively, 'split the bill' with your collaborator. Authors of fraudulent papers could be penalized by taking away from them, say, three tickets. Maybe we would even see the birth of a new class of ticket scalper and the production of counterfeit tickets. And if you are one of those extraordinarily persuasive scientists, you can always try to convince another researcher to give you one of their tickets so you can publish another influential piece of work.

The key question is: if you are unhappy with scientific publishing, would you agree to the 20 -paper limit? 